\title{
Continuous estimates on the earthquake early warning magnitude by use of the near-field acceleration records
}

\author{
Jun Li $\cdot$ Xing Jin • Yongxiang Wei • \\ Hongcai Zhang
}

Received: 29 August 2013/Accepted: 17 November 2013/Published online: 11 December 2013

(C) The Seismological Society of China, Institute of Geophysics, China Earthquake Administration and Springer-Verlag Berlin Heidelberg 2013

\begin{abstract}
In this article, the seismic records of Japan's Kik-net are selected to measure the acceleration, displacement, and effective peak acceleration of each seismic record within a certain time after $P$ wave, then a continuous estimation is given on earthquake early warning magnitude through statistical analysis method, and Wenchuan earthquake record is utilized to check the method. The results show that the reliability of earthquake early warning magnitude continuously increases with the increase of the seismic information, the biggest residual happens if the acceleration is adopted to fit earthquake magnitude, which may be caused by rich high-frequency components and large dispersion of peak value in acceleration record, the influence caused by the high-frequency components can be effectively reduced if the effective peak acceleration and peak displacement is adopted, it is estimated that the dispersion of earthquake magnitude obviously reduces, but it is easy for peak displacement to be affected by long-period drifting. In various components, the residual enlargement phenomenon at vertical direction is almost unobvious, thus it is recommended in this article that the effective peak acceleration at vertical direction is preferred to estimate earthquake early warning magnitude. Through adopting Wenchuan strong earthquake record to check the method
\end{abstract}

\footnotetext{
J. Li $(\bowtie) \cdot$ X. Jin $\cdot$ Y. Wei $\cdot$ H. Zhang

Earthquake Administration of Fujian Province, Fuzhou 350003,

China

e-mail: lixjix@163.com

X. Jin

Institute of Engineering Mechanics, China Earthquake

Administration, Harbin 150080, China

X. Jin

Fuzhou University, Fuzhou 350002, China
}

mentioned in this article, it is found that this method can be used to quickly, stably, and accurately estimate the early warning magnitude of this earthquake, which shows that this method is completely applicable for earthquake early warning.

Keywords Earthquake early warning · Near-field acceleration record . Effective peak value

\section{Introduction}

As an earthquake-prone country, China confronts a very severe situation in earthquake prevention and disaster reduction. Except for earthquake prediction and earthquake fortification, the earthquake early warning technology which makes use of travel time difference between $P$ wave and destructive seismic wave ( $\mathrm{S}$ wave or surface wave) as well as travel time difference between earthquake wave and electromagnetic wave is another effective means which can alleviate earthquake loss and reduce seismic secondary disaster. As a new prevention means for seismic hazard, the earthquake early warning has attracted the attention and been widely applied in many countries and regions. Currently, the seismic networks in partial regions in China have had the primary condition to construct earthquake early warning system (2008). The earthquake early warning technology has extremely high requirement for timeliness, the quick determination of earthquake parameter is a difficult point in earthquake early warning technology. As one of parameters representing three elements ("time, space, and strength") of earthquake, the earthquake magnitude is the quantitative description on the "size" and "strength" of earthquake in the earthquake early warning, we often use the earthquake magnitude to describe the size 
of earthquake. In current calculation of earthquake early warning magnitude, we mainly adopt empirical magnitude estimation methods by use of predominant period proposed by Nakamura $(1988,1989)$ and then developed by Allen and Kanamori (2003). Besides, we often use amplitude to measure and estimate the earthquake early warning magnitude. Grechsch and Kumpel (1997) use the record of acceleration at the 1 st second to estimate the magnitude, with an error of \pm 1.36 degree. Wu et al. $(1998,2007)$ and $\mathrm{Wu}$ and Zhao (2006) apply amplitude recorded within the initial $10 \mathrm{~s}$ to calculate $M_{\mathrm{L}} 10$ for nonlocal early warning and use the peak displacement from initial $\mathrm{P}$ waves $\left(P_{\mathrm{d}}\right)$ in magnitude determination for EEW. Zollo (2004, 2006a, b) makes use of the maximum value $P_{\mathrm{d}}$ of the initial displacement of $\mathrm{P}$ wave within $2 \mathrm{~s}$ of time window after $\mathrm{P}$ wave and the maximum value $S_{\mathrm{d}}$ of initial displacement of $\mathrm{S}$ wave within 1 and $2 \mathrm{~s}$ of time window after $\mathrm{S}$ wave to predict the magnitude. Ma (2008) combines amplitude with period method and makes use of neural network method to estimate the magnitude of earthquake. With time progress, the determination of earthquake early warning magnitude lacks continuous updating on earthquake magnitude. Therefore, it is very necessary to select a suitable method by which we can continuously analyze seismic oscillation information and continuously correct the estimated magnitude result according to the information about seismic wave received by seismograph station after the earthquake takes place. Meanwhile, in consideration that the main object of study in earthquake early warning is a major earthquake and there always exists amplitude limiting in near-field seismic measuring record when the major earthquake happens, it is also very necessary to make effective use of near-field strong earthquake record to estimate the earthquake early warning magnitude. This article selects strong earthquake record as research data for the hope of giving a real-time and continuous estimation on the earthquake early warning magnitude by use of observational data about strong earthquake.

\section{Introduction to information and method used}

Currently, there is only small quantity of nonsystematic data about major earthquake accumulated in China. Therefore, this article applies record about strong earthquake $\left(M_{\mathrm{j}} \geq 4.0\right)$ in underground basement by Japan's Kiknet from its establishment to August 2007. Kik-net refers to Japan's basement strong-motion observation network, each station is divided into pairing surface station and underground basement station, and most of wells have a depth of 100-200 m, with detailed geologic data (Aoi et al. 2004).

The main object of study in earthquake early warning research is the near-field major earthquake. Therefore, this
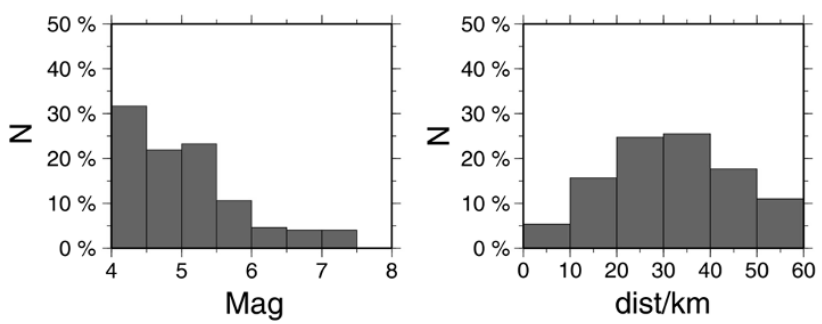

Fig. 1 Distribution of epicentral distance and earthquake magnitude

article selects the seismic record with a hypocentral distance of not more than $60 \mathrm{~km}$, makes selection for record according to signal-to-noise ratio (SNR), data discontinuance, data exception, and other information; and then picks the arrival time of $\mathrm{P}$ wave in each seismic record. The distribution of epicentral distance and the earthquake magnitude is shown in Fig. 1.

Generally, the earthquake magnitude is determined through measuring the amplitude of one seismic phase in seismic map, and the general form of magnitude scale is shown as below:

$M=\log \left(\frac{A}{T}\right)+f(\Delta, h)+C_{\mathrm{s}}+C_{\mathrm{r}}$

where, $M$ refers to the earthquake magnitude, $A$ refers to the amplitude of seismic oscillation used to determine the magnitude, $T$ refers to the corresponding period, $f(\Delta, h)$ refers to the correction factor used for the amplitude changing as the epicentral distance $\Delta$, and focal depth $h$, $C_{\mathrm{s}}$ refers to stylobate correction factors, and $C_{\mathrm{r}}$ refers to regional focus correction factor.

In the process of earthquake early warning, the earthquake early warning time is very short and we can only make use the limited initial record information recorded by limited seismic stations. Therefore, the determination of focal depth will have great error, and it is even impossible, thus the influence of focal depth will be not considered in this article. Besides, this article also gives no consideration on the difference between $\mathrm{S}$ wave and $\mathrm{P}$ wave, but directly measures and selects the maximum amplitude of all available information to estimate earthquake magnitude. We mainly consider the following two points:

(1) The rupture process of major earthquake has a long time of duration, and the near-field earthquake record is mutually affected by different rupture points, and it is hard to effectively distinguish $\mathrm{P}$ wave from $\mathrm{S}$ wave.

(2) We can regard seismic oscillation as the function of hypocentral distance, that is, the amplitude of the seismic oscillation is related to time after the triggering of $\mathrm{P}$ wave and epicentral distance. The epicentral distance will effect occurrence time of different seismic oscillation and attenuation of 


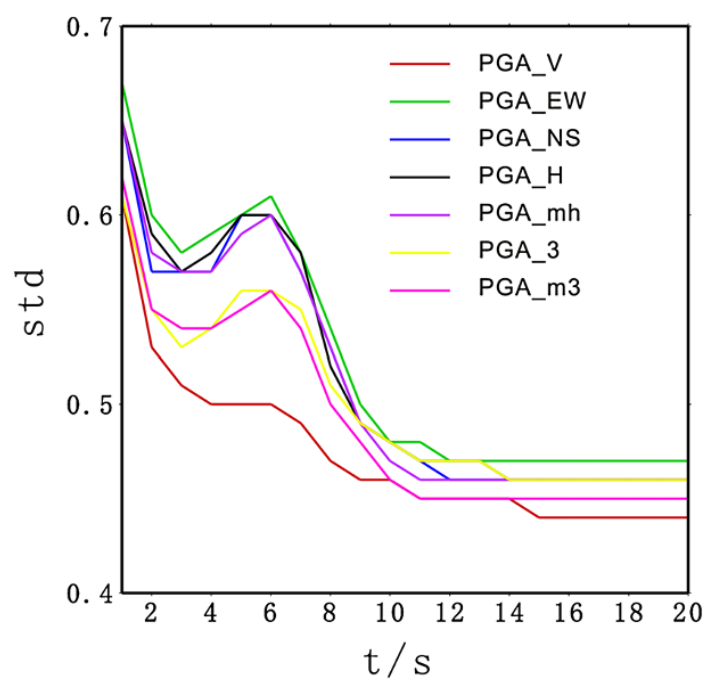

Fig. 2 Change of magnitude fitting residual as time

amplitude of seismic oscillation. Regardless of the influence of propagation path of seismic wave and stylobate, the amplitude of seismic oscillation is only related to the hypocentral (or epicentral) distance and earthquake magnitude at the same time after the triggering of $\mathrm{P}$ wave.

Through overall consideration, this article selects the magnitude estimation model as follows:

$M(t)=a(t) \log (A(t))+b(t) \log \left(\Delta+R_{0}\right)+c(t)$,

wherein, $A(t)$ refers to the maximum amplitude within $t$ second (s) after $\mathrm{P}$ wave, $\Delta$ is the epicentral distance, $R_{0}$ is the constant, $a(t), b(t), c(t)$ are the coefficients which need fitting. Through statistical analysis on the relationship between maximum amplitude and earthquake magnitude within $t$ second (s) after $\mathrm{P}$ wave in seismic record, the model is able to realize the continuous estimation on earthquake early warning magnitude.

\section{Continuous estimation on earthquake early warning magnitude by use of the acceleration record}

It is required to measure and select the peak acceleration within $t$ second (s) after $\mathrm{P}$ wave in each seismic record, and then make use of least square method to obtain the fitting formula (2). This article selects peak acceleration at vertical direction (PGA_V), peak acceleration at horizontal direction (PGA_EW, PGA_NS), peak resultant acceleration (PGA_H), average value of peak acceleration at horizontal direction (PGA_mh), peak three-component resultant acceleration (PGA_3), and average value of peak three-component acceleration (PGA_m3), and then gives a fitting for them, respectively. As shown in Fig. 2, the magnitude fitting residual of various peaks changes as the time.

The horizontal ordinate refers to the triggering time recorded by the station. It can be seen from the figure that the residual gradually decrease with the time progress, which shows that the earthquake early warning magnitude is increasingly reliable as there are more and more information about seismic oscillation. About at the 6th second, the residual increases; it is thought that the epicentral distance of the record selected is within $60 \mathrm{~km}$, about at the 6th second and it is estimated that the hypocentral distance is about $48 \mathrm{~km}$ based on a speed of imaginary wave of $8 \mathrm{~km} / \mathrm{s}$. At the time, there appears $\mathrm{S}$ wave in partial seismic record and only $\mathrm{P}$ wave is included in partial seismic record, there is no doubt that the fitting residual will increase if the information about $\mathrm{P}$ wave and the information about $\mathrm{S}$ wave are fitted together. There is small difference in residual among fitting results of different components, the best result is obtained at vertical direction, with almost unobvious residual enlargement.

If the peak acceleration is used to estimate the earthquake early warning magnitude, there is still large fitting residual. In view of many high-frequency components included in acceleration record and large dispersion, this article adopts the real-time simulation method proposed by Jin Xing et al. (2005) to simulate the seismic record as displacement record with a natural vibration period of $15 \mathrm{~s}$, and then makes a fitting again according to the formula (2). It is shown that the results of two kinds of peak fitting have a relatively consistent overall trend.

The earthquake early warning magnitude estimated by use of peak displacement has small dispersion. However, in view of the fact that the integral is needed twice in the process where the acceleration record is real-time simulated as displacement record, the displacement record obtained via simulation often has certain long-period drifting, and thus a high requirement is proposed for preprocessing of original acceleration record. This article hopes to directly measure and select one seismic oscillation value through acceleration record and further estimate earthquake magnitude. Meanwhile, this seismic oscillation shall monotonically increase or tend to become stable as the triggering time increases to avoid raising or lowering.

Through long-term discussion and practice, the researchers of Japan Meteorological Agency (JMA) formulate a set of complete instrument intensity calculation method. This method first makes filtering and combination for three-component record of seismic oscillation, and then selects the amplitude with duration of not less than $0.3 \mathrm{~s}$ during time interval of resultant acceleration as effective peak acceleration, Am. This method considers certain duration of seismic oscillation, and the effective peak obtained by this method can effectively reduce the 


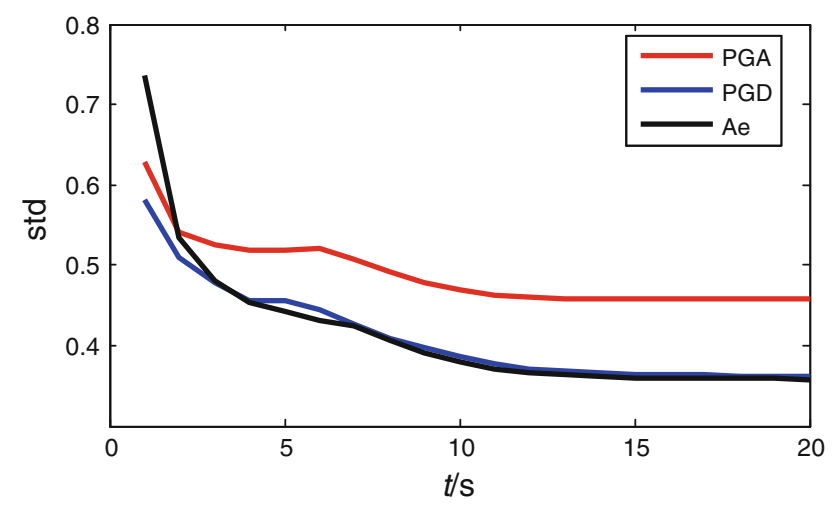

Fig. 3 Comparison diagram for prediction of magnitude by use of peak acceleration, effective peak acceleration, and peak displacement

discreteness brought by high-frequency components, and can also stably and accurately reflect the intensity of seismic oscillation.

With reference to this method, this article gives large quantity of statistics of relation between effective peak acceleration and duration and finds that the change of effective peak acceleration gradually tends to be gentle and that different seismic records have different rates of change with the increasing duration. Under the condition of comprehensive consideration on the stability of effective peak acceleration and timeliness of earthquake early warning, we select the amplitude with duration of not less than $0.5 \mathrm{~s}$ as effective peak acceleration and make a fitting again according to formula (2). Figure 3 refers to the comparison diagram for prediction of magnitude, respectively, by use of peak acceleration (PGA), effective peak acceleration (Ae), and peak displacement (PGD), all of them adopting vertical component. It can be seen from the figure that there is a small difference in dispersion of earthquake early warning magnitude, estimated by effective peak acceleration and peak displacement. We can see that the results of two kinds of peak fitting have a relatively consistent overall trend. The dispersion of magnitude estimated by use of peak acceleration is obviously larger than that estimated by use of peak displacement and effective peak acceleration. There will be about 0.1 magnitude difference in dispersion $10 \mathrm{~s}$ after triggering, and the reliability of magnitude estimation is obviously improved.

Figure 4 gives the fitting situation 3, 8, 15, and $20 \mathrm{~s}$ after triggering, the $x$-coordinate refers to actually measured magnitude, and the $y$-coordinate refers to the early warning magnitude. It can be seen that the dispersion of magnitude becomes smaller and smaller However, in the place with large magnitude, it seems that there is magnitude saturation phenomenon. It is thought via analysis that the minor earthquake has a large weight upon fitting due to few major earthquakes and many minor earthquakes in the earthquake sample, which will result in the situation that
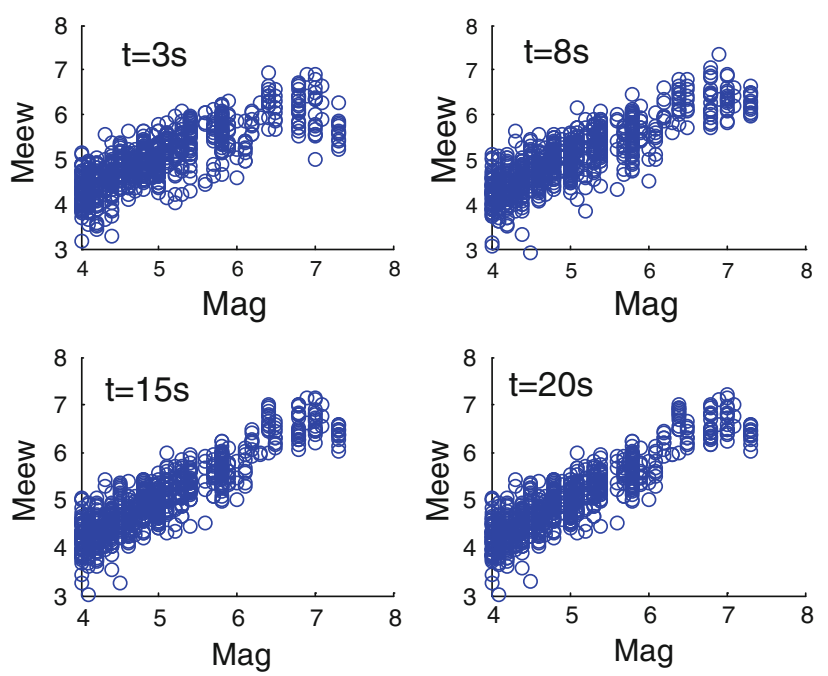

Fig. 4 Effect diagram for prediction of magnitude by use of effective peak acceleration

the major earthquake has a poorer determination effect of magnitude than the minor earthquake. The increase of weight of major earthquake upon fitting can improve the reliability of estimation on magnitude of major earthquake; However, it will also reduce reliability of estimation on magnitude of minor earthquake.

\section{Checking of Wenchuan earthquake record on the method}

The 8.0-magnitude strong earthquake record which happened on May 12, 2008 is selected to check the method mentioned in this article. Three stations (WCW, PXZ, and DXY) which are nearest to the epicenter are selected for analog calculation, with an epicentral distance of 21,36 , and $47 \mathrm{~km}$, respectively. The calculation result of early warning magnitude for each station is shown in Fig. 5. It can be seen that the magnitude is determined as greater than $6.2 \mathrm{~s}$ after $\mathrm{P}$ wave for three stations, which means that this method can be used to determine that this earthquake is a destructive earthquake $2 \mathrm{~s}$ after $\mathrm{P}$ wave. The earthquake early warning magnitude is continuously corrected and tends to be stable as the time progresses. Same as the above analysis, it is hard to effectively distinguish between $\mathrm{P}$ wave and $\mathrm{S}$ wave by the use of the near-field earthquake record due to long duration of rupture process of the major earthquake. Although there is no distinguishing between $\mathrm{P}$ wave and $\mathrm{S}$ wave in this method, there is no sudden jump in the magnitude in the simulation result of Wenchuan earthquake, which shows that this method is completely applicable for earthquake early warning. As for these three stations, the finally determined magnitude is about 7.5, which has certain gap with the actual 8.0. This may be 


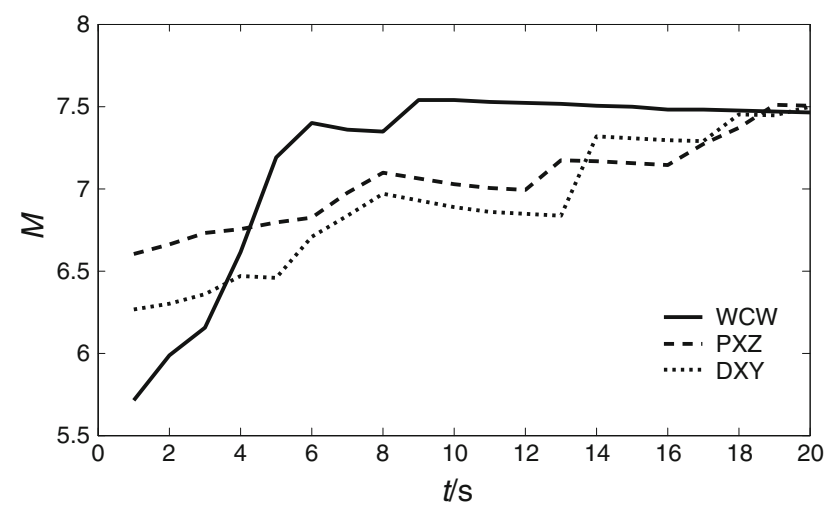

Fig. 5 Analog calculation result of Wenchuan earthquake

affected by magnitude saturation; thus, it is also very important to find a method which cannot be influenced by the magnitude saturation. Besides, it can be seen from the figure that the magnitude determined $10 \mathrm{~s}$ after $\mathrm{P}$ wave is not stable as for the stations YAJ and ZAT with slightly long epicentral distance, and this is also the reason why this article selects the fitting calculation of coefficients within the initial $20 \mathrm{~s}$.

\section{Results analysis}

This article selects 803 seismic records of Japan's Kik-net to measure and select the peak value within a certain time after $\mathrm{P}$ wave, and then continuously estimates the earthquake early warning magnitude through statistical analysis method. The result shows that the reliability of earthquake early warning magnitude continuously increases as the earthquake information increases, and there is the biggest residual if the peak acceleration is adopted to fit the magnitude, which may result from the rich high-frequency components and large dispersion of peak in acceleration record. The use of effective peak acceleration and peak displacement can effectively reduce the effect of highfrequency components, and the dispersion of magnitude estimated is obviously reduced, but peak displacement is easy for peak displacement to be affected by long-period drifting.

If three kinds of amplitudes are used to continuously estimate earthquake early warning magnitude, about at the 6th second, the residual increases, and it is thought that the epicentral distance of the record selected is within $60 \mathrm{~km}$, about at the 6th second. It is estimated that the hypocentral distance is about $48 \mathrm{~km}$ based on a speed of imaginary wave of $8 \mathrm{~km} / \mathrm{s}$. At this time, there appears $S$ wave in partial seismic record, and only $\mathrm{P}$ wave is included in partial seismic record. There is no doubt that the fitting residual will increase if the information about $\mathrm{P}$ wave and the information about $\mathrm{S}$ wave are fitted together. There is almost no obvious residual enlargement at vertical direction among various components. Therefore, it is recommended in this article that the effective peak acceleration at vertical direction is preferred to estimate the earthquake early warning magnitude.

Wenchuan strong earthquake record has been adopted to check the method mentioned in this article. The result shows that this method can quickly and accurately estimate the early warning magnitude of this earthquake. Although there is no distinguishing between $\mathrm{P}$ wave and $\mathrm{S}$ wave in this method, there is no sudden jump in the magnitude in the simulation result of Wenchuan earthquake, which shows that this method is completely applicable for earthquake early warning.

The epicentral distance is used in the process of fitting. There are always certain deviations in the earthquake early warning positioning. Therefore, a reliable earthquake early warning positioning result will be conducive to improving the reliability of this method which is used to estimate earthquake early warning magnitude. Besides, the records selected in this article all have an epicentral distance within $60 \mathrm{~km}$. Therefore, the fitting result in this article may not be applicable for earthquake with long epicenter.

This article selects Japan's seismic record for fitting, and the application of the obtained result in China may be limited to some extent. If there are enough seismic records, then we can obtain the calculation formula of earthquake early warning magnitude applicable for more regions through refitting.

In the calculation of effective peak acceleration, this article gives no filtering and other processing for the original record' The main reason is that the earthquake early warning has high requirements for timeliness; thus, the intermediate calculational process shall be reduced as much as possible, which will be conducive to reducing the processing time of earthquake early warning system and effectively improving the earthquake early warning time. If certain filtering processing is made for acceleration record to filter out partial high-frequency component, then it may be conducive to improving the reliability of earthquake early warning magnitude.

\section{References}

Allen RM, Kanamori H (2003) The potential for earthquake early warning in southern California. Science 300:786-789

Aoi S, Kunugi T, Fujiwara H (2004) Strong-motion seismograph network operated by NIED: K-NET and KiK-net. J Jpn Assoc Earthq Eng 4(3):65-74

Grecksch G, Kumpel HJ (1997) Statistical analysis of strong-motion accelerograms and its application to earthquake early-warning systems. Geophys J Int 129:113-123 
Ma Q (2008) Study and application on earthquake early warning [D]. Institute of engineering Mechanics China Earthquake Administration

Nakamura Y (1988) On the urgent earthquake detection and alarm system (UrEDAS). Proc Ninth World Conf Earthq Eng 7: 673-678

Nakamura Y (1989) Earthquake alarm system for Japan railways. Jpn Railw Eng 109:1-7

Wu YM, Kanamori H, Allen RM, Hauksson E (2007) Determination of earthquake early warning parameters, $\tau_{\mathrm{c}}$ and $\mathrm{P}_{\mathrm{d}}$, for southern California. Geophys J Int. doi:10.1111/J.1365-246X.2007.03430

Wu YM, Shin TC, Tsai YB (1998) Quick and reliable determination of magnitude for seismic early warning. Bull Seism Soc Am 88:1254-1259

Wu YM, Zhao L (2006) Magnitude estimation using the first three seconds p-wave amplitude in earthquake early warning. Geophys Res Lett 33:116312. doi:10.1029/2006GL026871
Xing Jin, Qiang Ma, Shanyou Li (2005) Real-time simulation of ground displacement by digital accelerograph record. Acta Seismol Sin 27(1):79-85

Zollo A, Iannaccone G, Manfredi G (2004) Development and experimentation of a prototype system for seismic early warning applications in Campania region (Southern Italy). Workshop on Seismic Early Warning for European Cities (Abstracts), 23-25 September 2004, Napoli, Italy

Zollo A, Lancieri M, Nielsen S (2006a) Earthquake magnitude estimation from peak amplitudes of very early seismic signals on strong motion records. Geophys Res Lett 33:L23312. doi:10. 1029/2006GL027795

Zollo A, Satriano C, Lancieri M, Lomax A, Bobbio A, Cantore L, Convertito (2006b) Real-time estimation of earthquake location and magnitude for seismic early warning in Campania region, southern Italy. Seismol Res Lett 77(2):313-314 